\title{
Protective distal side-to-side neurorrhaphy in proximal nerve injury-an experimental study with rats
}

\author{
Henrikki Rönkkö ${ }^{1}$ (D) $\cdot$ Harry Göransson ${ }^{2} \cdot$ Hanna-Stiina Taskinen ${ }^{3} \cdot$ Pasi Paavilainen $^{2} \cdot$ Tero Vahlberg $^{4} \cdot$ Matias Röyttä $^{5}$
}

Received: 25 November 2018 / Accepted: 31 January 2019 /Published online: 12 February 2019

(C) The Author(s) 2019

\begin{abstract}
Background Side-to-side neurorrhaphy may protect the denervated end organ and preserve the initial connection with proximal stump. We examined the effect of protective side-to-side anastomosis on nerve and end organ regeneration in proximal nerve injury model.

Methods The left common peroneal nerve of 24 Sprague Dawley rats was proximally transected. In groups B and C, side-to-side neurorrhaphy was performed distally between the peroneal and tibial nerves without (group B) and with (group C) partial donor nerve axotomy inside the epineural window. Group A served as an unprotected control. After 26 weeks, the proximal transection was repaired with end-to-end neurorrhaphy on all animals. Regeneration was followed during 12 weeks with the walk track analysis. Morphometric studies and wet muscle mass calculations were conducted at the end of the follow-up period.

Results The results of the walk track analysis were significantly better in groups B and C compared to group A. Groups B and C showed significantly higher wet mass ratios of the tibialis anterior and extensor digitorum longus muscle compared to group A. Group C showed significantly higher morphometric values compared to group A. Group B reached higher values of the fibre count, fibre density, and percentage of the fibre area compared to group A.

Conclusions Protective distal side-to-side neurorrhaphy reduced muscle atrophy and had an improving effect on the morphometric studies and walk track analysis. Distal side-to-side neurorrhaphy does not prevent the regenerating axons to grow from the proximal stump to achieve distal nerve stump.
\end{abstract}

Keywords Peripheral nerves $\cdot$ Nerve injury $\cdot$ Chronic denervation $\cdot$ Nerve repair $\cdot$ Nerve regeneration $\cdot$ Side-to-side repair

This article is part of the Topical Collection on Peripheral Nerves

Henrikki Rönkkö

henrikki.ronkko@tuni.fi

1 Department of Orthopaedics, Kanta-Häme Central Hospital, Hämeenlinna, Finland

2 Department of Hand and Microsurgery, Tampere University Hospital, Tampere, Finland

3 Department of Hand Surgery, Turku University Hospital, Turku, Finland

4 Institute of Clinical Medicine, Department of Biostatistics, University of Turku, Turku, Finland

5 Department of Pathology, University of Turku, Turku, Finland

\section{Introduction}

The regenerative results have often been unsatisfactory in proximal nerve injuries. This has been considered a consequence of the degenerative changes of the nerve due to the delay of the growing axons to reach their end organs. The number of Schwann cells begins to decline, and their capacity to secrete neurotrophins decreases [15, 21, 27, 29, 38]. Basal lamina tubes begin to degrade and will be substituted with connective tissue $[25,35]$. At the same time, muscle atrophy proceeds because of lack of reinnervation.

It has been presumed that timely reinnervation of the distal nerve and end organ could solve the problem. Distal end-toend (ETE) $[2,9,22,33,34,37]$ and end-to-side (ETS) $[3,4,7$, $8,12,16-18,31,32]$ nerve transfers and free nerve grafts $[14$, $20,36]$ have been used.

Distal side-to-side (STS) anastomosis has been shown to have regenerative potential clinically in the treatment of 
proximal nerve injuries $[6,39,40]$ However, to our knowledge, there are no experimental comprehensive studies dealing with side-to-side anastomosis in proximal nerve injuries in international literature. This method leaves the distal nerve free for further reconstructions. It is potentially advantageous, especially in cases where regeneration from the proximal stump of the injured nerve is expected. In addition, the repair can be performed in one operation and there is no need for nerve grafts. This systematic experimental study was conducted to examine the ability of the distal side-to-side nerve anastomosis to give time to proximal regenerating nerve to grow distally avoiding degeneration of the distal nerve and muscle. In our model, delayed end-to-end repair was used to simulate the proximal injury.

We hypothesise that protective distal side-to-side anastomosis has an improving effect on the results of walk track analysis and nerve morphometry and reduces muscle atrophy related to proximal nerve injury. We also presume that intentional donor nerve axotomy inside side-to-side anastomosis improves the repair results.

\section{Materials and methods}

\section{Animals}

Twenty-four female young adult Sprague Dawley rats (Central Animal Laboratory, University of Turku, Finland) weighing 223-300 g were randomly divided into three experimental groups. The animals were allowed to receive laboratory chow and drink tap water ad libitum. The temperature was kept at $22{ }^{\circ} \mathrm{C} \pm 2{ }^{\circ} \mathrm{C}$, and the humidity was $50 \% \pm 10 \%$. The day cycle in the animal room was constant (lights on from 6:00 AM to 6:00 PM). There were no wound infections during the experiment. The National Animal Experiment Board approved all interventions, the analgesic treatment, and animal care (ESLH200901886/Ym-23, the decision STH168A).

\section{Operative procedure}

In the first operation under medetomidine hydrochloride $5 \mu \mathrm{g} / \mathrm{kg}$ (Domitor, Orion Oyj, Espoo, Finland) and ketamine hydrochloride $750 \mu \mathrm{g} / \mathrm{kg}$ (Ketalar, Pfizer Oy, Helsinki, Finland) anaesthesia, the left common peroneal nerve (CPN) was ligated with two sequential 8-0 sutures (Nylon, S\&T AG, Neuhausen, Switzerland) $5 \mathrm{~mm}$ distally from the site of bifurcation of the left common peroneal nerve and tibial nerve (TN) on all animals (Fig. 1). Transection of the common peroneal nerve was done between the ligations, and the nerve ends were turned in opposite directions and sutured in the adjacent muscle with three 10-0 sutures (Nylon, S\&T AG, Neuhausen, Switzerland) to prevent reinnervation. In group B, an additional distal side-to-side anastomosis was performed with 2-mm-long epineural windows in both common peroneal nerve and tibial nerve and sutured with four 11-0 microsurgical nylon knots (Monosof, Covidien, Mansfield, MA, USA) under surgical microscopy Wild M3Z (Wild Leitz Ltd., Heerbrugg, Switzerland). In group $\mathrm{C}$, the operation was similar to group B, but in distal sideto-side anastomosis, an axotomy to the extent of half of the donor tibial nerve was performed. Group A served as an unprotected control. In all animals, the muscle layer and skin were closed in separate layers with 5-0 absorbable sutures (Bondek Plus, Deknatel, TFX Medical Ltd., High Wycombe, UK). Perioperatively, the animals were injected subcutaneously $5 \mathrm{ml}$ sodium chloride $9 \mathrm{mg} / \mathrm{ml}$ (Fresenius Kabi AB, Uppsala, Sverige) to maintain fluid balance. A subcutaneous injection $5 \mathrm{mg} / \mathrm{kg}$ carprofen (Rimadyl, Vericode Ltd., Dundee, UK) was given to animals during three postoperative days as an analgesic drug.

After 26 weeks, a second operation was performed on all animals. Normal appearance, construction, and consistence of nerve ends were ensured by recurrent sharp excisions of neuroma formations. Proximal injury of common peroneal nerve was repaired with tension-free end-to-end anastomosis with four 11-0 microsurgical nylon knots (Monosof, Covidien, Mansfield, MA, USA) under surgical microscopy. The same investigator carried out all operations. The follow-up time was 12 weeks after the second operation.

\section{Walk track analysis}

The walk track analysis was performed preoperatively, and $2,4,6,8,12,16,20$, and 26 weeks after the first operation, and $2,4,6,8$, and 12 weeks after second operation. The animals walked freely at least six times per time point in a steady velocity along an $80 \times 115 \times 515$-mm corridor with a darkened box at the end. Only walks without stops were included in analysis. Ink staining from both hind limbs was analysed. The print length (PL, distance between heel and third toe), toe spread (TS, distance between first and fifth toe), and intermediate toe spread (IT, distance between second and fourth toe) were calculated as a mean of three measurements. The peroneal function index (PFI) was calculated with following formula: PFI $=174.9((E P L-N P L) /$ NPL $)+80.3(($ ETS - NTS $) / N T S)-13.4$ [1]. "N" refers to the normal, unoperated side, and "E" refers to the experimental side The investigator has passed the self-education test of the walk track analysis [5] and was blinded to know the intervention groups during analysis.

\section{Sample preparation}

Terminal anaesthesia was induced with an intraperitoneal injection of sodium pentobarbital $60 \mathrm{mg} / \mathrm{kg}$ (Mebunat, 
Fig. 1 Schematic representation of interventions. In the first operation (upper panel), in all groups, the common peroneal nerve $(\mathrm{CPN})$ is proximally transected and left unrepaired. In group B, distal side-to-side (STS) neurorrhaphy is performed without intentional axonal injury to the nerves. In group $\mathrm{C}$, donor axonal injury to the extent of half of the nerve is done to the donor nerve inside the epineural windows and side-to-side anastomosis is performed similarly to the previous group. After 26 weeks, the second operation (lower panel) is performed similarly in all groups; proximal injury is repaired in an end-to-end fashion. The red marks show the nerve biopsy sites
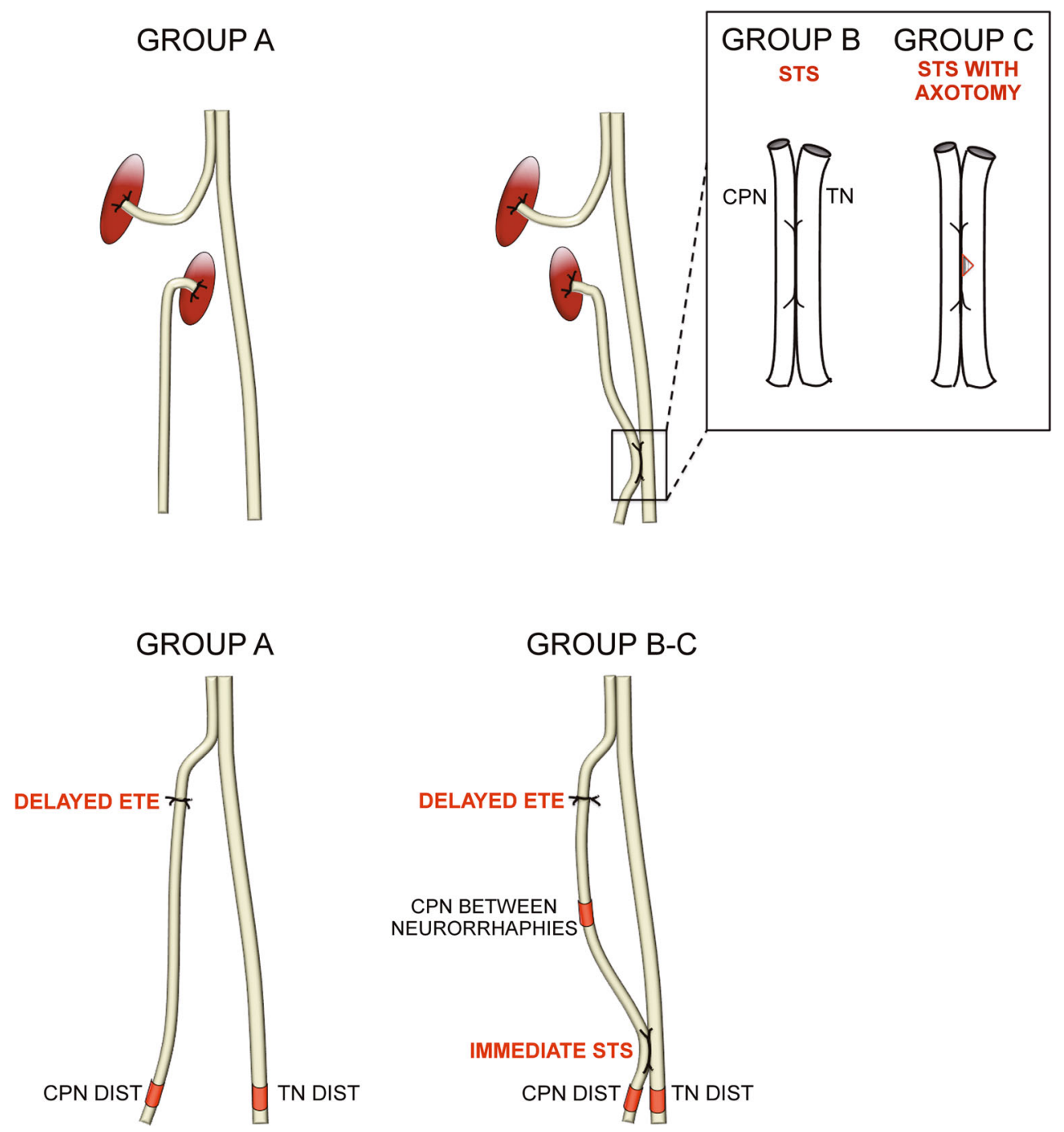

Orion Oyj, Espoo, Finland). After perfusion of formalin, operated nerves were harvested. The sites of nerve samples of common peroneal nerve and tibial nerve taken to the morphometric analysis are shown in Fig. 1. Common peroneal nerve-innervated tibial anterior and extensor digitorum longus and tibial nerve-innervated gastrocnemius muscles were carefully dissected from both legs with the microsurgical technique and weighed with a balance PG403-S DeltaRange (Mettler-Toledo GmbH, Greifensee, Switzerland). Tissue samples were immersion-fixed in phosphate-buffered formalin for overnight. Fourmicrometre-thick sections were cut from paraffin blocks for haematoxylin and eosin staining or immunocytochemistry.

\section{Neurofilament protein immunocytochemistry}

The stainings were performed with a biotin-free Poly-HRPAnti-Mouse kit (BrightVision, Immunologic BV, Duiven, The Netherlands) according to the manufacturer's instructions. Mouse monoclonal neurofilament $(200 \mathrm{kDa}$ and 68 kDa) Ab1 (Clone 2F11) antibody (Thermo Fisher Scientific, Fremont, CA, USA) was applied and incubated. Normal Antibody Diluent (Immunologic BV, Duiven, The Netherlands) was used to dilute and stabilise HRP-conjugates. The sections were incubated with peroxidasecompatible chromogen (Bright-DAB, Immunologic BV, Duiven, The Netherlands) for $8 \mathrm{~min}$ and finally counterstained and coverslipped.

\section{Morphometry}

Neurofilament-stained whole-nerve cross-sections were photographed with an AxioCam HRc camera (Carl Zeiss, Göttingen, Germany) connected to an AxioVert $200 \mathrm{M}$ microscope (Carl Zeiss, Göttingen, Germany). The mosaic images were adjusted with AxioVision software (Carl Zeiss, Jena, Germany). Imaging software (Graphics Suite X6/PhotoPaint, Corel Comp., Ottawa, ON, Canada) was used to process 
subepineural areas of digitalized images. With BioImageXD software [19], the following morphometric outcomes were analysed: nerve area $\left(\mu \mathrm{m}^{2}\right)$, fibre count, fibre area $\left(\mu \mathrm{m}^{2}\right)$, total fibre area $\left(\mu \mathrm{m}^{2}\right)$, fibre density $\left(\mathrm{n} / \mathrm{mm}^{2}\right)$, and percentage of fibre area (total fibre area/nerve area $\times 100$; Table 1 ).

\section{Statistical analysis}

The statistical analysis was done with the help of an experienced statistician with SPSS software (version 24, IBM Corp., Armonk, NY, USA). In our earlier study, the sideto-side repair group with donor axotomy reached a peroneal function index value of 35.7 (9.08) at 12 weeks postoperatively. The sample size of eight animals per group gives $90 \%$ power and a type I error rate of no more than 5\% to detect a difference of 15 or more in the mean peroneal function index values between groups. Differences with $p<0.05$ were considered statistically significant. The data are expressed in terms of mean (SD).

Type 3 tests of fixed effects were used to reveal significant differences between the intervention groups. The comparisons between the groups in the results of the walk track analysis were analysed with the covariance analysis ANCOVA for repeated measurements which was adjusted to the baseline peroneal function index values. Inside every group, the value before second operation and the value at the end of the follow-up period were compared to reveal the effect of second operation. The Tukey-Kramer adjustment was used to control the effect of multiple comparisons.
In the morphometric analysis, the groups were compared with the one-way ANOVA variance analysis with TukeyKramer adjustment for multiple comparisons. If the global $p$ value was significant, we have made pairwise comparisons between groups using Tukey's method. Comparison of two different biopsy sites of the same nerve was performed with the paired $t$ test. The fibre area values were normally distributed after $\log 10$-transformation. The linear mixed model with Tukey-Kramer and Dunnett adjustments was used to compare the nerve fibre area values.

The wet mass ratios were compared using the MannWhitney $U$ test with Bonferroni adjustment for multiple comparisons. The correlations between the peroneal function index, wet mass ratios, and morphometric outcomes were calculated with Pearson correlation coefficients.

\section{Results}

\section{Walk track analysis}

Groups $\mathrm{B}$ and $\mathrm{C}$ reached significantly higher peroneal function index values compared to group A from 8 weeks onwards to the end of the follow-up time (Fig. 2). Groups B and C did not differ. When comparing the peroneal function index values at 26 weeks before the second operation to the peroneal function index values at the end of the follow-up, there were significant increases in all groups (group A, $p<0.001 ; \mathrm{B}, p=0.007$; and $\mathrm{C}, p=0.008$ ).

Table 1 Results of morphometric analyses of common peroneal nerve and tibial nerve

\begin{tabular}{|c|c|c|c|c|c|c|}
\hline & Nerve area $\left(\mu \mathrm{m}^{2}\right)$ & Fibre count & $\begin{array}{l}\text { Mean fibre } \\
\text { area }\left(\mu \mathrm{m}^{2}\right)\end{array}$ & $\begin{array}{l}\text { Total fibre } \\
\text { area }\left(\mu \mathrm{m}^{2}\right)\end{array}$ & $\begin{array}{l}\text { Fibre density } \\
\left(\mathrm{n} / \mathrm{mm}^{2}\right)\end{array}$ & $\begin{array}{l}\text { Percentage } \\
\text { of fibre area }(\%)\end{array}$ \\
\hline \multicolumn{7}{|c|}{ Common peroneal nerve } \\
\hline \multicolumn{7}{|c|}{ Between neurorrhaphies } \\
\hline Group B & $58,047(19618)$ & $1613(468)$ & $3.7(0.50)$ & $6100(2208)$ & $29,044(6616)$ & $10.7(2.3)$ \\
\hline Group C & $77,098(26166)$ & $2810(711)$ & $4.0(0.35)$ & $11,236(3078)$ & $37,395(4354)$ & $14.9(2.8)$ \\
\hline \multicolumn{7}{|l|}{ Distal } \\
\hline Group A & $29,497(15773)$ & 353 (239) & $3.0(0.66)$ & $1088(856)$ & $13,242(9176)$ & $4.0(2.9)$ \\
\hline Group B & $57,434(14409)$ & $1573(305)$ & $4.5(0.80)$ & $7042(1875)$ & $27,869(3714)$ & $12.3(1.2)$ \\
\hline Group C & $88,926(38971)$ & $3044(706)$ & $5.4(0.59)$ & $16,277(5175)$ & $36,819(7280)$ & $19.1(3.4)$ \\
\hline Intact control & $148,517(26881)$ & $2313(102)$ & $20.3(2.7)$ & $46,998(7461)$ & $15,943(2310)$ & $31.9(3.4)$ \\
\hline \multicolumn{7}{|l|}{ Tibial nerve } \\
\hline \multicolumn{7}{|l|}{ Distal } \\
\hline Group A & 436,961 (104329) & $5527(278)$ & $20.0(4.6)$ & $110,704(27063)$ & $13,165(2575)$ & $25.4(3.2)$ \\
\hline Group B & $295,626(49259)$ & $5234(488)$ & $13.9(2.0)$ & $71,962(6849)$ & $17,951(2149)$ & $24.8(4.0)$ \\
\hline Group C & $244,208(57916)$ & $4973(651)$ & $10.9(2.2)$ & $53,692(9747)$ & $20,963(3665)$ & $22.3(2.8)$ \\
\hline Intact control & $372,928(84512)$ & $5212(500)$ & $20.8(2.7)$ & $108,870(21318)$ & $14,409(2323)$ & $29.4(2.2)$ \\
\hline
\end{tabular}

Data is expressed in terms of mean (SD) 


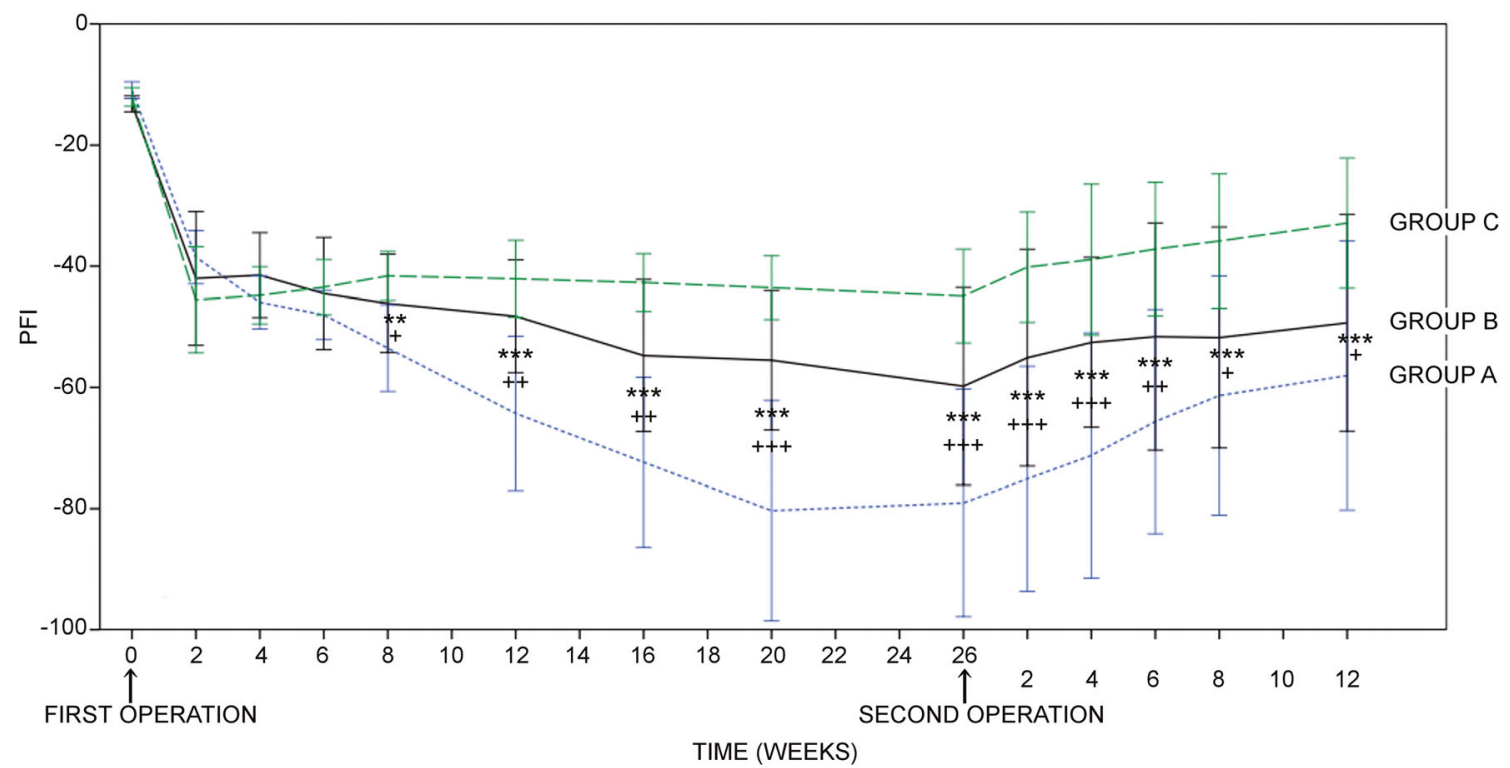

Fig. 2 Results of the walk track analysis. Groups B and C show significantly higher peroneal function index (PFI) values compared to group A from 8 weeks onward. There are no significant differences between groups B and C. The data are analysed with the analysis of

\section{Morphometry}

\section{Common peroneal nerve (Table 1)}

Distal sections of common peroneal nerve (Fig. 1, lower panel) All morphometric outcomes except mean fibre area of group $\mathrm{C}$ showed significantly higher values compared to group $\mathrm{A}($ all $p<0.001$ ) (Fig. 3a-f). In group $\mathrm{B}$, the fibre count, fibre density, and percentage of the fibre area reached significantly higher values compared to group A. The fibre count, total fibre area, fibre density, and percentage of the fibre area values were higher in group $\mathrm{C}$ compared to group B (all $p \leq 0.03)$. The intervention groups did not reach the nerve area, total fibre area, and percentage of the fibre area values of the intact group (all $p<0.001)$. The fibre count and fibre density values of group $\mathrm{C}$ showed higher values compared to the intact group (both $p \leq 0.004$ ). The mean fibre area values of the intact group were higher compared to three intervention groups (all $p<0.001$ ). There were no significant differences between the groups $\mathrm{A}, \mathrm{B}$, and $\mathrm{C}$.

The specimens between neurorrhaphies (Fig. 1, lower panel) Group C showed higher values of fibre count, total fibre area, fibre density, and percentage of the fibre area compared to group B. Groups B and C reached higher values of all morphometric outcomes compared to the distal specimens of group A (all $p \leq 0.03$ ). covariance with Tukey-Kramer adjustment for multiple comparisons. $* p<0.05, * * p<0.01, * * * p<0.001$ comparison of group $\mathrm{A}$ to $\mathrm{C}$, ${ }^{+} p<0.05,{ }^{++} p<0.01,{ }^{++} p<0.001$ comparison of group A to B. Error bar, $\pm 1 \mathrm{SD}$

When comparing two different biopsy sites, the total fibre area, and the percentage of the fibre area values of group $\mathrm{C}$ were higher in the distal sections compared to the sections between the neurorrhaphies (both $p \leq 0.04$ ). In group B, the morphometric values of two biopsy sites did not differ significantly.

\section{Tibial nerve (Table 1)}

Distal specimens of tibial nerve were studied (Fig. 1, lower panel). The fibre count and the percentage of the fibre area values of the intervention groups did not differ significantly. The mean fibre area and total fibre area values of group A were higher compared to group $\mathrm{C}$ (both $p<0.001$ ) but not to group B. Group A did not differ compared to the intact group in any morphometric outcome. The mean fibre area, total fibre area, and percentage of the fibre area values of the intact group were significantly higher compared to groups B and $\mathrm{C}$ (all $p<0.04)$.

\section{Light microscopy}

Distal common peroneal nerve In group $\mathrm{A}$, both the nerve area and the individual axons looked smaller compared to the other groups, and in some sections, there were connective tissue in the subepineural area (Fig. 4). 

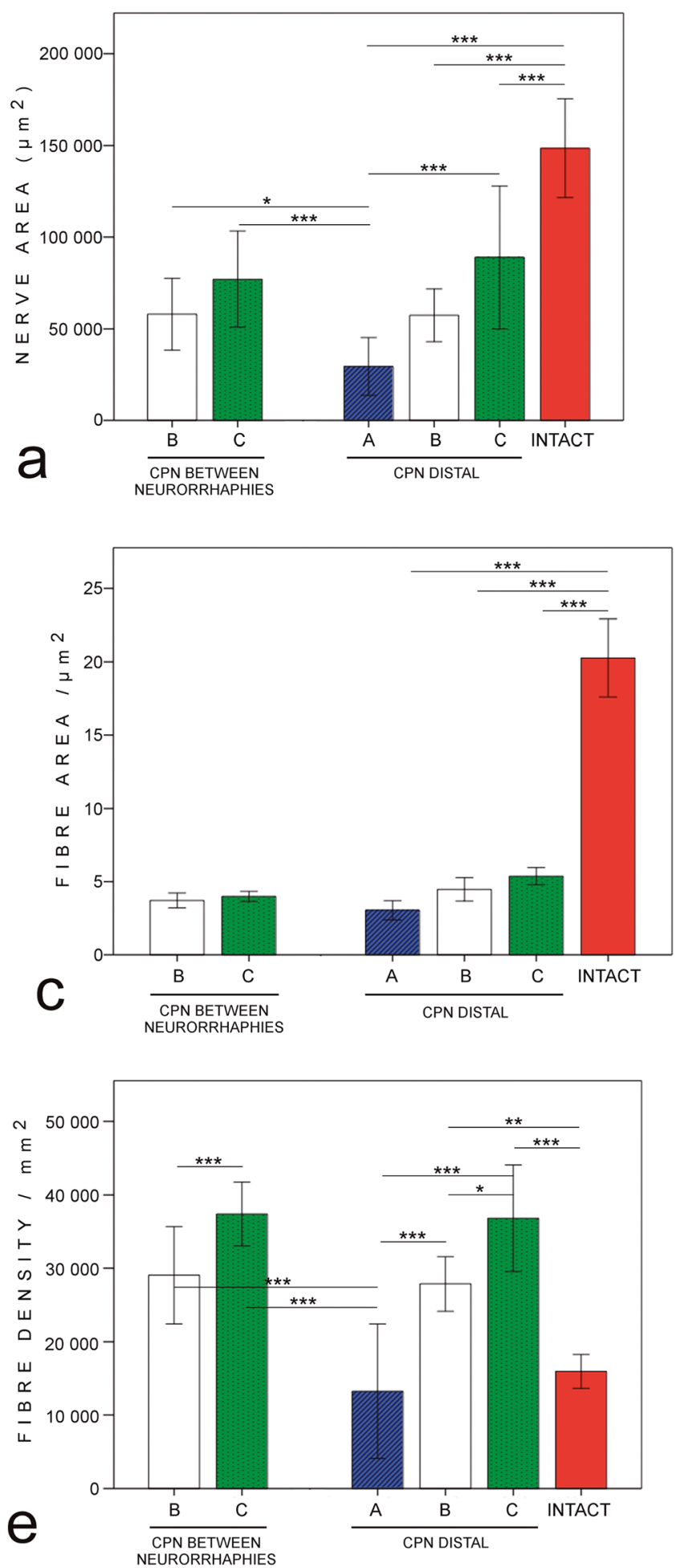

Fig. 3 Morphometric results of the common peroneal nerve. Group C shows statistically significantly higher values of all morphometric outcomes except mean fibre area compared to group A. Group B reaches higher values of fibre count (b), fibre density (e), and percentage of the fibre area (f) compared to group A. When comparing

Common peroneal nerve between neurorrhaphies The axonal regeneration was more robust in group $\mathrm{C}$ compared to group
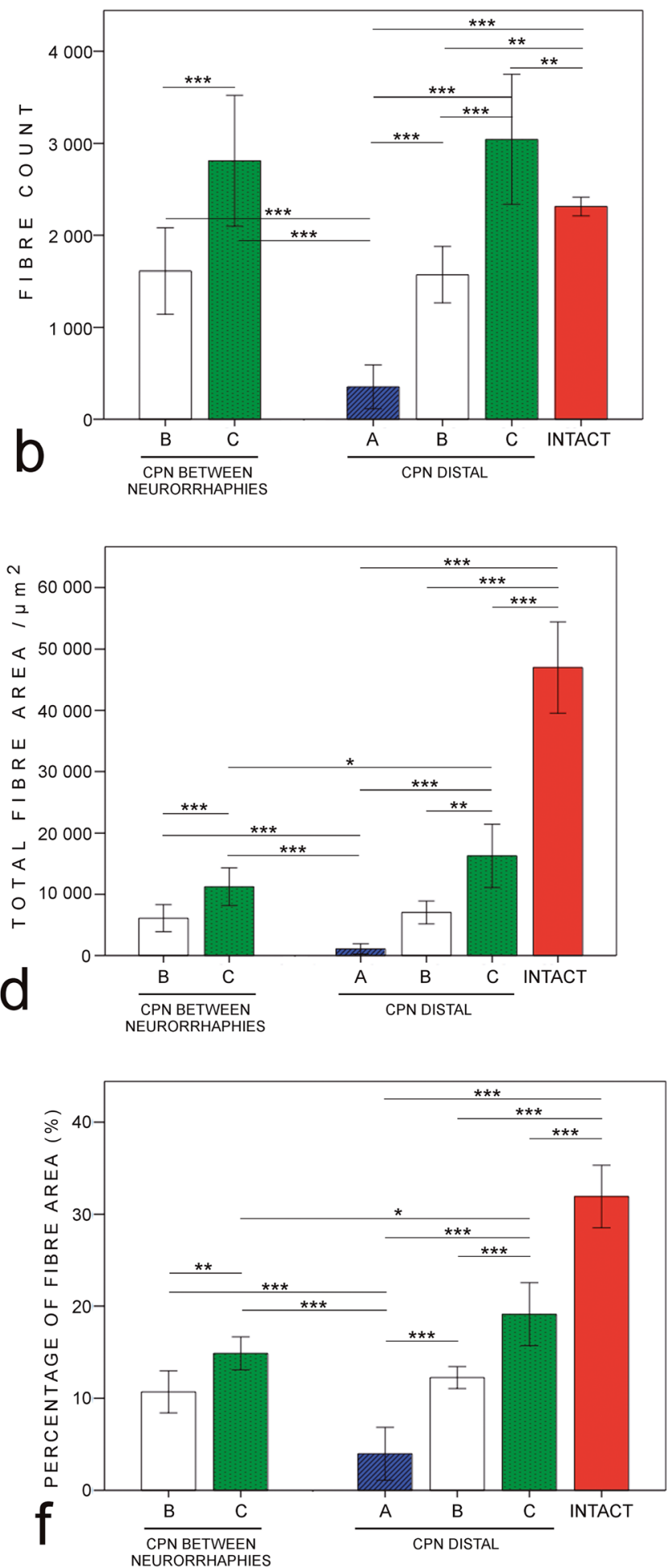

groups $\mathrm{B}$ and $\mathrm{C}$, group $\mathrm{C}$ shows higher values of fibre count (b), total fibre area (d), fibre density (e), and percentage of the fibre area (f) both at the site between neurorrhaphies and distal to common peroneal nerve. $* p<0.05, * * p<0.01, * * * p<0.001$. Bars express the mean values, error bar $\pm 1 \mathrm{SD}$

B. In both groups, some axon sprouts were seen outside the epineurium. 


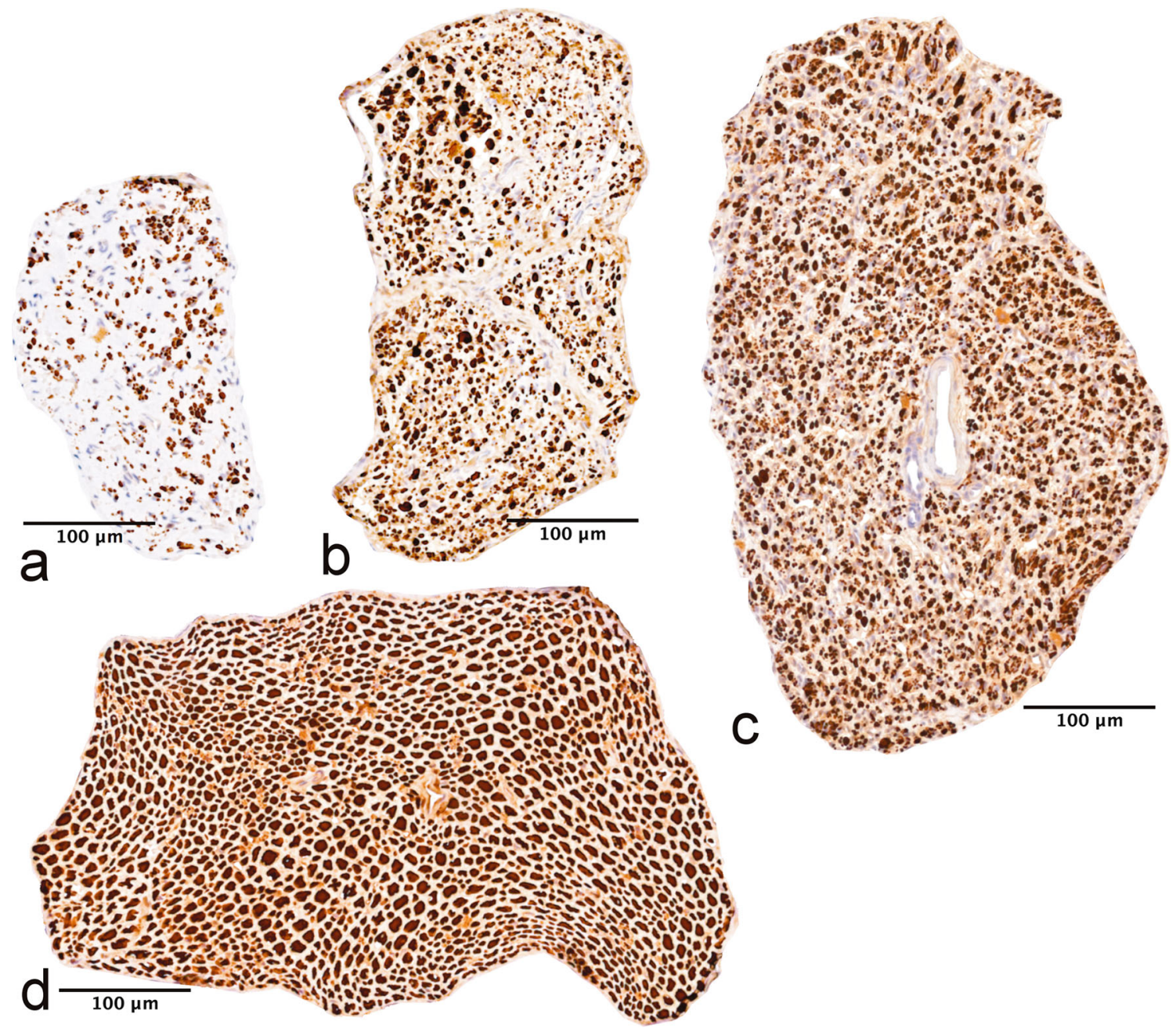

Fig. 4 Nerve sections of the distal common peroneal nerve. Morphometric analyses are done with whole-nerve cross-sections. The nerve structure is better preserved in groups B (b) and C (c) compared to group A (a). In groups B and C, the fibre density is high, but the fibre area is smaller compared to intact nerve (d). Neurofilament staining
Distal tibial nerve In group $\mathrm{A}$, the sections looked normal. Occasionally, in groups $\mathrm{B}$ and $\mathrm{C}$, some axons were seen outside the epineurium. In some sections of group $\mathrm{C}$, the axons were smaller in the lateral zones.

Tibialis anterior and extensor digitorum longus muscles In group A, there were clear signs of general atrophy with small, angular-shaped cells. In groups B and C, the size and shape of muscle cells were generally normal.

Gastrocnemius muscle In all groups, the general appearance was normal, but in groups B and C, mild focal signs of atrophy were detected.

\section{Muscle mass calculations}

In groups $\mathrm{B}$ and $\mathrm{C}$, the wet mass ratio of the tibialis anterior muscle (Fig. 5) and extensor digitorum longus muscle (Fig. 6) were significantly higher compared to group A (both $p<0.001$ ). The wet mass ratio of the gastrocnemius muscle (Fig. 7) was significantly higher in group A compared to group $\mathrm{C}(p<0.05)$, whereas there were no significant differences between groups $\mathrm{A}$ and $\mathrm{B}$ and groups $\mathrm{B}$ and $\mathrm{C}$.

\section{Correlations between outcomes}

The morphometric parameters of distal common peroneal nerve, the wet muscle ratios of the tibialis anterior and extensor digitorum longus muscles, and the corresponding peroneal function index values of the walk track analysis at the end of the follow-up period correlated significantly with each other. Morphometric parameters of distal common peroneal nerve correlated with peroneal function index value at 12 weeks: nerve area (Pearson correlation $0.41, p=0.05$ ), fibre count $(0.63, p<0.001)$, fibre area $(0.54, p=0.006)$, total fibre area 
Fig. 5 HE-stained biopsies and macroscopic figures of the tibialis anterior muscle of the intervention groups $(\mathbf{a}-\mathbf{c})$ and control sample from the contralateral side (d). In group A (a), the muscle fibres are small and angular-shaped (asterisk). In groups B and C, focal signs of atrophy can be seen. The wet mass ratio (e) is significantly higher in groups $\mathrm{B}$ and $\mathrm{C}$ compared to group A. In group A, the wet muscle mass value of tibialis anterior (TA) muscle is 22 (6.2)\% of the contralateral side value. In groups $\mathrm{B}$ and $\mathrm{C}$, the values are 54 (9.6)\% and $62(8.5) \%$, respectively. $* p<0.05$, ** $p<0.01$, $* * * p<0.001$. Bars express the mean values, error bar $\pm 1 \mathrm{SD}$
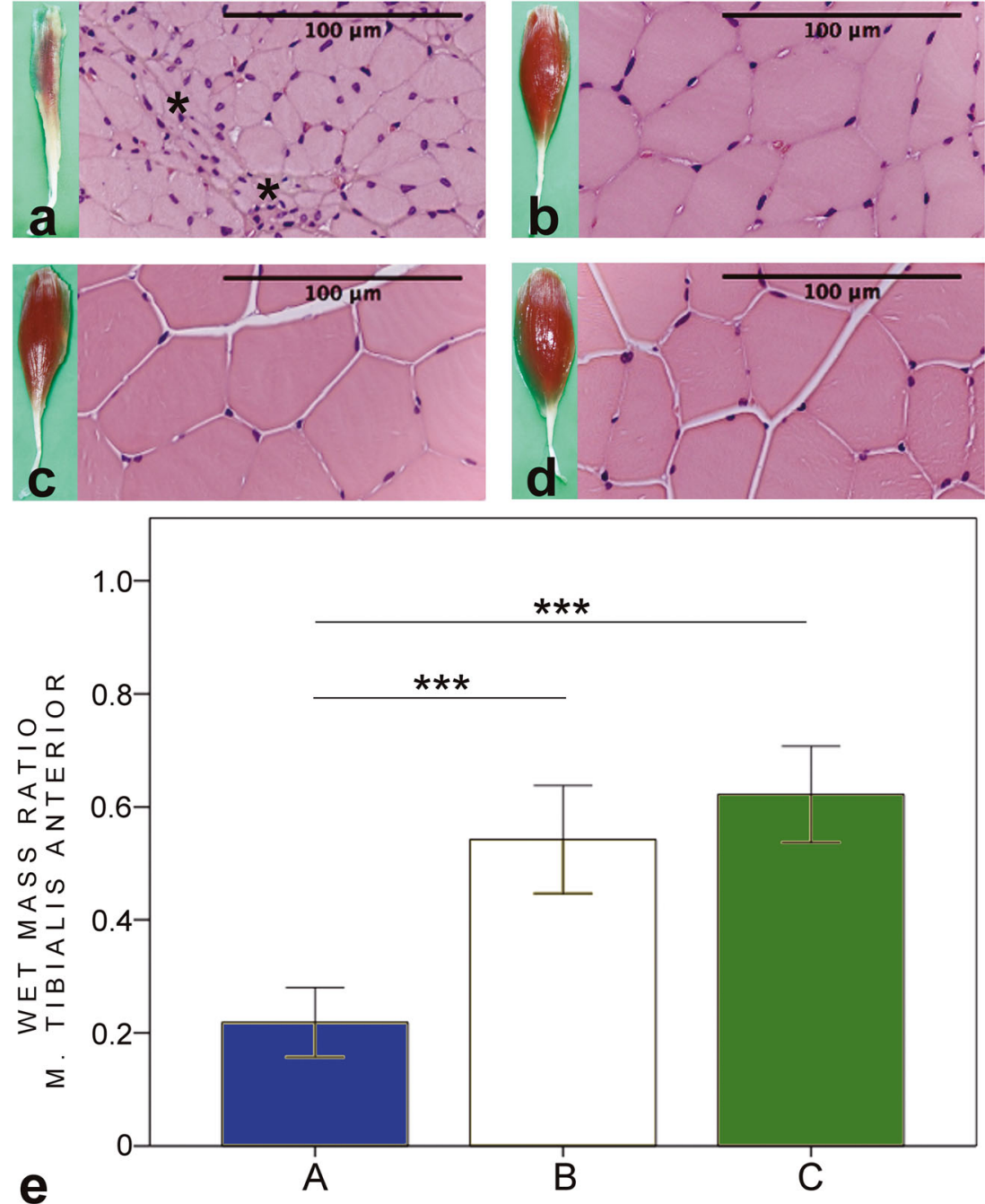

$(0.57, p=0.003)$, fibre density $(0.74, p<0.001)$, and percentage of fibre area $(0.71, p<0.001)$. Significant correlations were found also with wet muscle ratios of tibialis anterior and extensor digitorum longus muscles: nerve area (both Pearson correlations $\geq 0.55$, both $p \leq 0.005$ ), fibre count $(\geq$ $0.78, p<0.001)$, fibre area $(\geq 0.78, p<0.001)$, total fibre area $(\geq 0.73, p<0.001)$, fibre density $(\geq 0.80, p<0.001)$, and percentage of fibre area $(\geq 0.89, p<0.001)$. Peroneal function index value at 12 weeks correlated with wet mass ratios of tibialis anterior and extensor digitorum longus muscles (both $\geq 0.65, p<0.001)$.

\section{Discussion}

The regeneration capacity of the proximal peripheral nerve injury is a multilevel problem. The success of proximal nerve repair depends both on the capacity of regenerating axons to arrive to distal stump [23] and the capacity of the distal nerve stump to support the neural regeneration before muscle atrophy occurs. Fu and Gordon $[10,11]$ showed that prolonged denervation of Schwann cells in the distal stump is more detrimental to nerve regeneration than prolonged axotomy of the proximal stump. Furthermore, in case of proximal injury, the repair can always be considered delayed due to the lengthened time it takes to reach the end organs.

In the present study, immediate distal side-to-side neurorrhaphy near the end organs was performed to reduce muscle atrophy and to maintain the growth-supportive environment in the distal nerve stump. Protective distal side-toside anastomosis (groups B and C) had an improving effect on the results of the morphometric studies, muscle wet mass, and walk track analysis (PFI) compared to unprotected delayed end-to-end nerve repair (group A).

In morphometry, the parameters of both side-to-side groups differed significantly compared to unprotected end-to-end repair. In our previous study, side-to-side repair without deliberate axotomy produced mean 881 fibres to the distal common peroneal nerve at 26 weeks representing the innervation of immediate side-to-side anastomosis to 
Fig. 6 HE-stained biopsies and macroscopic figures of the extensor digitorum longus muscle of the intervention groups $(\mathbf{a}-\mathbf{c})$ and control sample from the contralateral side (d). Clear signs of atrophy and replacement of adipose tissue (asterisk) are seen in group $\mathrm{A}$. In groups $\mathrm{B}$ and $\mathrm{C}$, the muscle cells are large, but there are focal signs of atrophy. The wet mass ratio (e) is significantly higher in groups B and C compared to group A. In group A, the wet muscle mass value of extensor digitorum longus (EDL) muscle is $26(5.2) \%$, in group B 80 (9.3)\%, and in group C 86 (10.1)\% of the contralateral side values. $* p<0.05, * * p<0.01$, $* * * p<0.001$. Bars express the mean values, error bar $\pm 1 \mathrm{SD}$
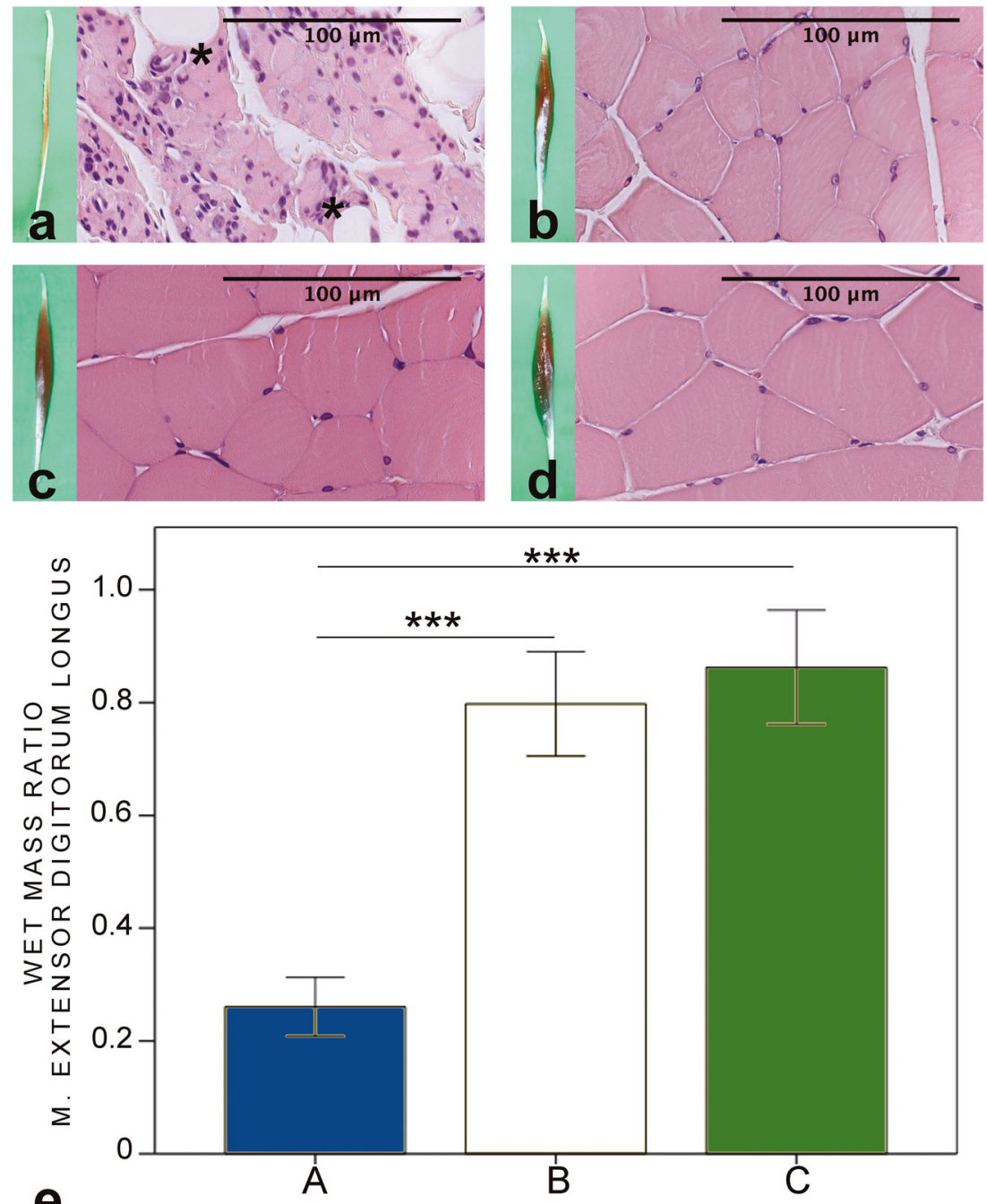

the recipient nerve [24]. In the present study, there were mean 1573 fibres in the distal common peroneal nerve in group B representing the combined innervation of both the immediate side-to-side anastomosis and delayed proximal end-to-end repair. In group A, the fibre count was mean 353 after mere delayed proximal end-to-end repair. Thus, it can be assumed that the immediate side-to-side anastomosis did not prevent the ingrowth of the regenerating axons from the proximal end to reach the distal nerve stump after delayed end-to-end repair. The mechanism controlling axonal regeneration in distal nerve stump is not totally understood. We know that without regeneration, the denervated Schwann cells change their phenotype from growthsupportive to myelinating progressively after 1 month $[15,21,38]$. Exogenous cytokines have been used to mimic interactions between macrophages and Schwann cells and to restore the growth-promoting capacity of Schwann cells $[13,28]$. In the side-to-side bridge model, Schwann cells in the denervated recipient nerve are shown to be able to change their phenotype after reconstruction to a proliferative phenotype and again to redifferentiate to a myelinating phenotype when the donor axon has grown into the recipient nerve [14].

In groups $\mathrm{B}$ and $\mathrm{C}$, the wet mass ratios of the common peroneal nerve-innervated tibialis anterior, and the extensor digitorum longus muscles, were significantly higher compared to group A. Distal side-to-side anastomosis could clearly reduce muscle atrophy. Our result is in relation to the study of Ladak et al. [11]. They reported with 4-month delayed repair a 1.6-fold increase in the tibialis anterior wet muscle weight at a 5-month follow-up compared to the unprotected group [20]. In our study, the increase was 2.5-fold. Although the fibre count values were significantly higher in group $\mathrm{C}$ when compared to group B, the wet mass ratios between the groups did not differ. Additional donor nerve axotomy inside side-to-side anastomosis (group C) did not significantly increase the recipient wet mass ratios compared to side-to-side neurorrhaphy without donor axonal injury (group B). A 
Fig. 7 HE-stained biopsies and macroscopic figures of the gastrocnemius muscle of the intervention groups $(\mathbf{a}-\mathbf{c})$ and control sample from the contralateral side (d). Muscle architecture was well preserved in all groups. However, mild focal signs of atrophy were detected in groups $\mathrm{B}$ and $\mathrm{C}$. In wet mass ratio calculations (e), group A (89 (6.0) $\%$ ) got higher values compared to group C (82 (7.8) \%). Groups B $(83(4.9) \%)$ and $\mathrm{C}$ did not differ. $* p<0.05, * * p<0.01$,

$* * * p<0.001$. Bars express the mean values, error bar $\pm 1 \mathrm{SD}$
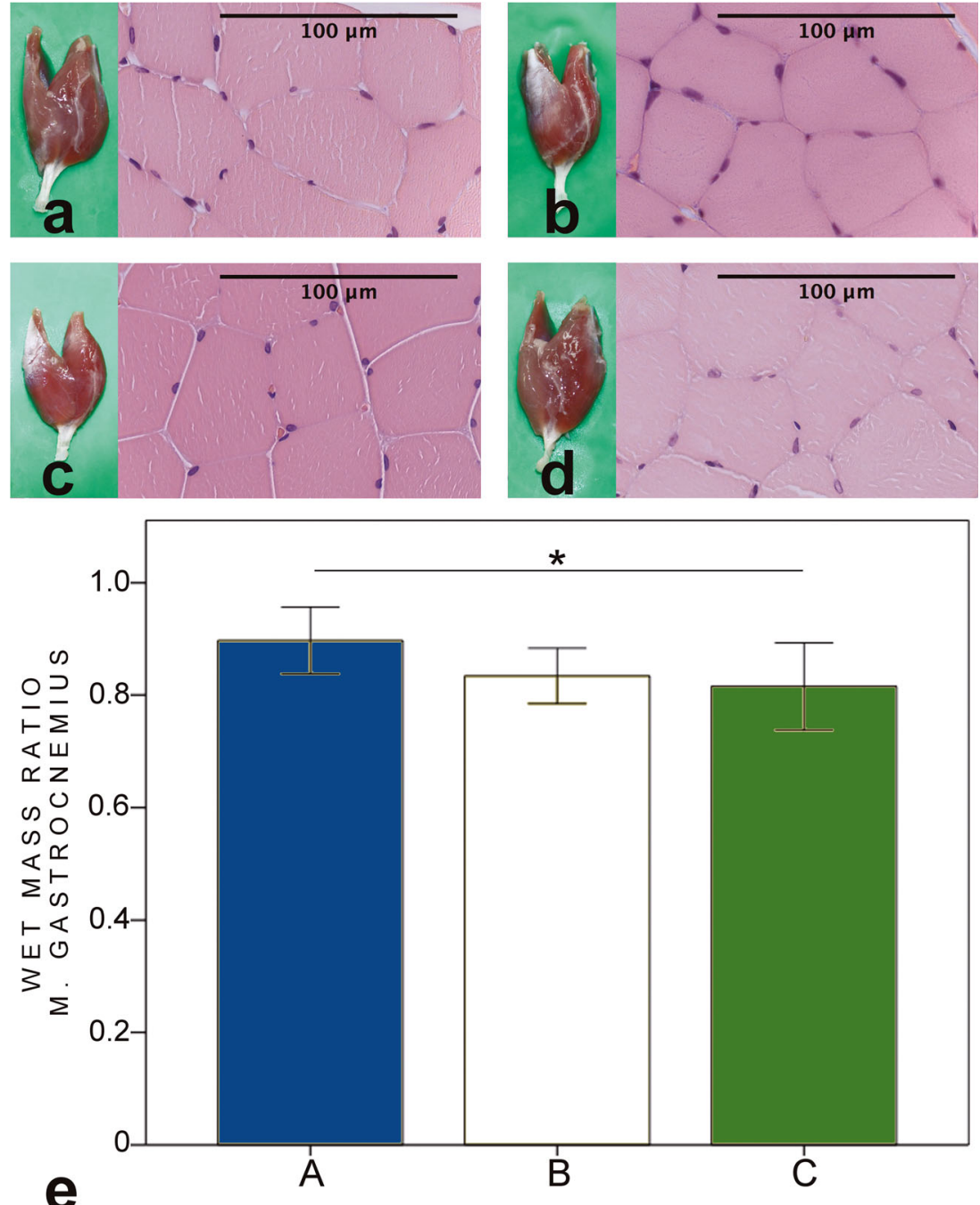

different number of axons preserved muscle mass at the similar level. It is known that denervated muscle fibres can partly compensate the reduced number of motor units by increasing the size of motor units $[11,30]$. Denervated muscle fibres are able to receive reinnervation to some extent, but they are not able to recover to the earlier diameter [11]. This was seen in the present study with delayed end-to-end repair (group A), as the peroneal function index values improved with limited axonal flow, but the muscle atrophy remained considerable. The tibialis anterior muscle wet mass ratio (0.22) of group A was equal compared to unrepaired group after 32 weeks denervation ( 0.21 , our unpublished data).

The results of the walk track analysis showed that in groups $\mathrm{B}$ and $\mathrm{C}$, the recovery was twofold. The first improvement was seen after the distal side-to-side repair and the second after the delayed proximal end-to-end repair. This suggests that both the immediate side-to-side repair and the delayed proximal end-toend repair have influence on the functional outcome, and thus, both repair procedures are worth performing. The results are in accordance with the previous so-called baby-sitting techniques $[3,4,7,8,12,16-18,31,32]$.

When evaluating the effects of partial donor nerve axotomy on recipient nerve, also, the effects on donor nerve have to be assessed. In the present study, side-toside with mere epineural window did not have significant deleterious effect on donor nerve. However, the impairing effect was seen with side-to-side protection with deliberate partial donor nerve axotomy. The mean fibre area and total fibre area values were higher in the unprotected group (group A) compared to the side-to-side protection with donor nerve axotomy (group C). Also, the muscle mass ratio of gastrocnemius muscle was higher in unprotected group (group A, 89.7\%) compared to side-to-side protection with donor nerve axotomy (group C, 81.5\%) (Fig. 7). The side-to-side protection with epineural window and unprotected group did not have significant differences in the results of donor nerve morphometry or wet muscle mass ratios. 
Distal side-to-side anastomosis may provide a useful tool to reconstruct proximal complete or incomplete nerve injuries. It does not sacrifice the distal end of the injured nerve or a healthy adjacent nerve. However, a prerequisite for this technique is the appropriate anatomical structure that allows the approximation of two parallel nerve trunks. For cases not allowing this, various other techniques have been developed to connect parallel nerves to the side-to-side bridge technique with nerve grafts $[14,20,36]$ or synthetic conduits [26]. The obvious weaknesses of the present study are the differences in the regeneration capacity as well as the differences of the nerves between rodents and humans. In the morphometric analysis, the regeneration of motor axons could not be separated from sensory axons, and the neurofilament staining does not allow the measurement of myelin sheath. In further studies, retrograde labelling techniques and electron microscopy are warranted.

\section{Conclusion}

The effect of early distal side-to-side anastomosis was studied in the proximal nerve injury model. Distal sideto-side neurorrhaphies were able to reduce muscle atrophy. Side-to-side anastomosis with partial axotomy improved the morphometric results, but did not have a significant effect on the recipient nerve innervating muscle mass amounts or the results of the walk track analysis compared to bare side-to-side anastomosis. Protective distal side-toside neurorrhaphies did not impair axon growth from the proximal stump to the distal nerve after delayed proximal end-to-end neurorrhaphy. Both immediate side-to-side anastomosis and delayed end-to-end repairs contributed to the improved results of the walk track analysis. This technique may have potential to improve the results in high-level nerve injuries.

Acknowledgements Mrs. Sinikka Collanus and other laboratory staff of the University of Turku are gratefully acknowledged.

Funding Funding was not received from any foundation requiring open access. Henrikki Rönkkö has received financial support for this study from the following foundations: the Medical Research Fund of Turku University Hospital, the Medical Research Fund of Tampere University Hospital, and the Finnish Society for Surgery of Hand. The sponsors had no role in the design or conduct of this research.

\section{Compliance with ethical standards}

Conflict of interest The authors declare that they have no conflict of interest.

Ethical approval All procedures performed in studies involving animals were in accordance with the ethical standards of the institution or practice at which the studies were conducted. This article does not contain any studies with human participants performed by any of the authors.

Open Access This article is distributed under the terms of the Creative Commons Attribution 4.0 International License (http:// creativecommons.org/licenses/by/4.0/), which permits unrestricted use, distribution, and reproduction in any medium, provided you give appropriate credit to the original author(s) and the source, provide a link to the Creative Commons license, and indicate if changes were made.

Publisher's note Springer Nature remains neutral with regard to jurisdictional claims in published maps and institutional affiliations.

\section{References}

1. Bain JR, Mackinnon SE, Hunter DA (1989) Functional evaluation of complete sciatic, peroneal, and posterior tibial nerve lesions in the rat. Plast Reconstr Surg 83:129-138

2. Bain JR, Veltri KL, Chamberlain D, Fahnestock M (2001) Improved functional recovery of denervated skeletal muscle after temporary sensory nerve innervation. Neuroscience 103:503-510

3. Baltzer HMD, Woo AMD, Oh CMD, Moran SLMD (2016) Comparison of ulnar intrinsic function following supercharge end-to-side anterior interosseous-to-ulnar motor nerve transfer: a matched cohort study of proximal ulnar nerve injury patients. Plast Reconstr Surg 138:1264-1272

4. Barbour J, Yee A, Kahn LC, Mackinnon SE (2012) Supercharged end-to-side anterior interosseous to ulnar motor nerve transfer for intrinsic musculature reinnervation. J Hand Surg Am 37:2150 2159

5. Brown CJ, Mackinnon SE, Evans PJ, Bain JR, Makino AP, Hunter DA, Hare GM (1989) Self-evaluation of walking-track measurement using a sciatic function index. Microsurgery 10:226-235

6. Cage TA, Simon NG, Bourque S, Noss R, Engstrom JW, Ralph JW, Kliot M (2013) Dual reinnervation of biceps muscle after side-toside anastomosis of an intact median nerve and a damaged musculocutaneous nerve. J Neurosurg 119:929-933

7. Davidge KMMD, M.S.C, Yee ABS, Moore AMMD, Mackinnon SEMD (2015) The supercharge end-to-side anterior interosseousto-ulnar motor nerve transfer for restoring intrinsic function: clinical experience. Plast Reconstr Surg 136:344e-352e

8. Farber SJ, Glaus SW, Moore AM, Hunter DA, Mackinnon SE, Johnson PJ (2013) Supercharge nerve transfer to enhance motor recovery: a laboratory study. J Hand Surg Am 38:466-477

9. Fox IKMD, Davidge KMMD, M.S., Novak CBPD, Hoben GMD, Kahn LCBS, Juknis NMD, Ruvinskaya RMD, Mackinnon SEMD (2015) Nerve transfers to restore upper extremity function in cervical spinal cord injury: update and preliminary outcomes. Plast Reconstr Surg 136:780-792

10. Fu SY, Gordon T (1995) Contributing factors to poor functional recovery after delayed nerve repair: prolonged axotomy. J Neurosci 15:3876-3885

11. Fu SY, Gordon T (1995) Contributing factors to poor functional recovery after delayed nerve repair: prolonged denervation. J Neurosci 15:3886-3895

12. Fujiwara T, Matsuda K, Kubo T, Tomita K, Hattori R, Masuoka T, Yano K, Hosokawa K (2007) Axonal supercharging technique using reverse end-to-side neurorrhaphy in peripheral nerve repair: an experimental study in the rat model. J Neurosurg 107:821-829

13. Gordon T, Sulaiman O, Boyd JG (2003) Experimental strategies to promote functional recovery after peripheral nerve injuries. $\mathrm{J}$ Peripher Nerv Syst 8:236-250 
14. Hendry JM, AlvarezVeronesi MCMAS, SnyderWarwick A, Gordon T, Borschel GH (2015) Side-to-side nerve bridges support donor axon regeneration into chronically denervated nerves and are associated with characteristic changes in Schwann cell phenotype. Neurosurgery 77:803-813

15. Hoke A, Gordon T, Zochodne DW, Sulaiman OA (2002) A decline in glial cell-line-derived neurotrophic factor expression is associated with impaired regeneration after long-term Schwann cell denervation. Exp Neurol 173:77-85

16. Isaacs J, Allen D, Chen LE, Nunley J 2nd (2005) Reverse end-toside neurotization. J Reconstr Microsurg 21:43-48

17. Isaacs JE, Cheatham $\mathrm{S}$, Gagnon EB, Razavi A, McDowell CL (2008) Reverse end-to-side neurotization in a regenerating nerve. J Reconstr Microsurg 24:489-496

18. Kale SS, Glaus SW, Yee A, Nicoson MC, Hunter DA, Mackinnon SE, Johnson PJ (2011) Reverse end-to-side nerve transfer: from animal model to clinical use. J Hand Surg Am 36:1631-1639.e2

19. Kankaanpaa P, Paavolainen L, Tiitta S, Karjalainen M, Paivarinne J, Nieminen J, Marjomaki V, Heino J, White DJ (2012) BioImageXD: an open, general-purpose and high-throughput image-processing platform. Nat Methods 9:683-689

20. Ladak A, Schembri P, Olson J, Udina E, Tyreman NBS, Gordon T (2011) Side-to-side nerve grafts sustain chronically denervated peripheral nerve pathways during axon regeneration and result in improved functional reinnervation. Neurosurgery 68:1654-1666

21. Li H, Terenghi G, Hall SM (1997) Effects of delayed re-innervation on the expression of c-erbB receptors by chronically denervated rat Schwann cells in vivo. Glia 20:333-347

22. Ray WZ, Chang J, Hawasli A, Wilson TJ, Yang L (2016) Motor nerve transfers: a comprehensive review. Neurosurgery 78:1-26

23. Ronkko H, Goransson H, Siironen P, Taskinen HS, Vuorinen V, Roytta M (2011) The capacity of the distal stump of peripheral nerve to receive growing axons after two and six months denervation. Scand J Surg 100:223-229

24. Ronkko H, Goransson H, Taskinen H, Paavilainen P, Vahlberg T, Roytta M (2016) Comparison of peripheral nerve regeneration with side-to-side, end-to-side, and end-to-end repairs: an experimental study. Plast Reconstr Surg Glob Open 4:e1179

25. Roytta M, Salonen V (1988) Long-term endoneurial changes after nerve transection. Acta Neuropathol 76:35-45

26. Shea JE, Garlick JW, Salama ME, Mendenhall SD, Moran LA, Agarwal JP (2014) Side-to-side nerve bridges reduce muscle atrophy after peripheral nerve injury in a rodent model. J Surg Res 187 : 350-358

27. Sulaiman OA, Gordon T (2009) Role of chronic Schwann cell denervation in poor functional recovery after nerve injuries and experimental strategies to combat it. Neurosurgery 65:A105-A114

28. Sulaiman OA, Gordon T (2002) Transforming growth factor-beta and forskolin attenuate the adverse effects of long-term Schwann cell denervation on peripheral nerve regeneration in vivo. Glia 37: 206-218

29. Sulaiman OA, Gordon T (2000) Effects of short- and long-term Schwann cell denervation on peripheral nerve regeneration, myelination, and size. Glia 32:234-246

30. Sulaiman OA, Midha R, Munro CA, Matsuyama T, Al-Majed A, Gordon T (2002) Chronic Schwann cell denervation and the presence of a sensory nerve reduce motor axonal regeneration. Exp Neurol 176:342-354

31. Terzis JK, Tzafetta K (2009) "Babysitter" procedure with concomitant muscle transfer in facial paralysis. Plast Reconstr Surg 124: $1142-1156$

32. Terzis JK, Tzafetta K (2009) The "babysitter" procedure: minihypoglossal to facial nerve transfer and cross-facial nerve grafting. Plast Reconstr Surg 123:865-876

33. Tung TH, Mackinnon SE (2010) Nerve transfers: indications, techniques, and outcomes. J Hand Surg Am 35:332-341

34. Veltri KMS, Kwiecien JMDVM, Minet WBS, Fahnestock MPD, Bain JRMS (2005) Contribution of the distal nerve sheath to nerve and muscle preservation following denervation and sensory protection. J Reconstr Microsurg 21:57-70

35. Vuorinen V, Siironen J, Röyttä M (1995) Axonal regeneration into chronically denervated distal stump. 1. Electron microscope studies. Acta Neuropathol 89:209-218

36. Wan H, Zhang L, Li D, Hao S, Feng J, Oudinet JP, Schumacher M, Liu S (2014) Hypoglossal-facial nerve "side"-to-side neurorrhaphy for persistent incomplete facial palsy. J Neurosurg 120:263-272

37. Willand MPPD, Holmes MBS, Bain JRMD, de Bruin HPD, Fahnestock MPD (2014) Sensory nerve cross-anastomosis and electrical muscle stimulation synergistically enhance functional recovery of chronically denervated muscle. Plast Reconstr Surg 134: $736 \mathrm{e}-745 \mathrm{e}$

38. You S, Petrov T, Chung PH, Gordon T (1997) The expression of the low affinity nerve growth factor receptor in long-term denervated Schwann cells. Glia 20:87-100

39. Yuksel F, Peker F, Celikoz B (2004) Two applications of end-toside nerve neurorrhaphy in severe upper-extremity nerve injuries. Microsurgery 24:363-368

40. Zhang S, Ji F, Tong D, Li M (2012) Side-to-side neurorrhaphy for high-level peripheral nerve injuries. Acta Neurochir 154:527-532

\section{Comments}

This article is very well planned, executed, and written. It demonstrates the beneficial effect of performing a distal side-to-side nerve anastomosis with or without a partial axotomy, in the setting of a proximal nerve injury that is repaired directly in a delayed manner. It has important clinical implications.

Michel Kliot

CA, USA 